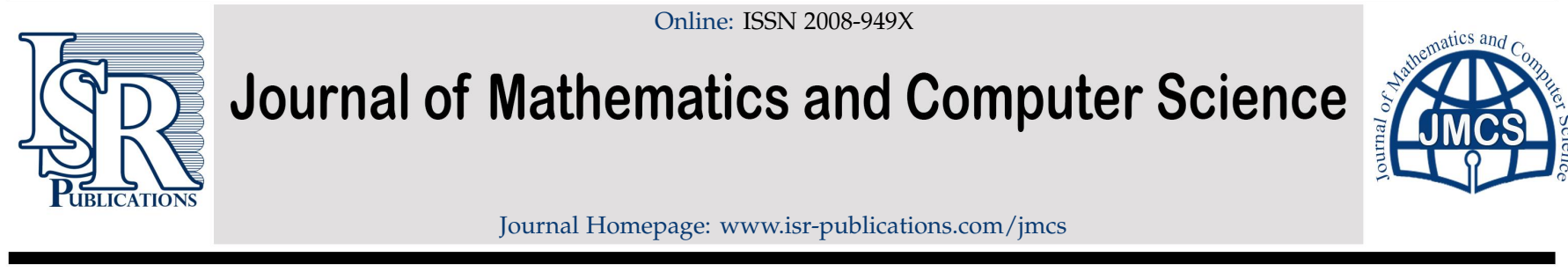

\title{
Solution of fractional autonomous ordinary differential equations
}

\author{
Rami AlAhmad ${ }^{\mathrm{a}, \mathrm{c}, *}$, Qusai AlAhmad $^{\mathrm{b}}$, Ahmad Abdelhadic \\ a Mathematics department, Yarmouk University, Irbid, 21163, Jordan. \\ ${ }^{b}$ Mathematics Department, California state university at Northridge, Northridge, CA 91330-8313, USA. \\ ${ }^{c}$ Department of Mathematics and Natural Sciences, Higher colleges of technology, Ras AlKhaimah, UAE.
}

\begin{abstract}
Autonomous differential equations of fractional order and non-singular kernel are solved. While solutions can be obtained through numerical, graphical, or analytical solutions, we seek an implicit analytical solution.
\end{abstract}

Keywords: Fractional derivatives, Caputo fractional derivative, the Caputo-Fabrizio fractional derivative, Laplace transform. 2020 MSC: 44A10, 26A33.

(C)2022 All rights reserved.

\section{Introduction}

Fractional calculus has resurfaced and gained momentum due to its potential in engineering systems, multidisciplinary fields, biology, medicine, and applied sciences. Its wide range of applications includes areas like linear anomalous diffusion equation and its characteristics [12], modeling biological phenomena, respiratory tissue, and drug diffusion [13]. At the same time, fractional calculus has found its way to sensors, analog filters and digital filters [14].

Solving differential equations plays a major role in Engineering, Physics, Biology, and other fields like economics and medicine, see [4-6]. Consider the autonomous ordinary differential equations

$$
\frac{d y}{d t}=F(y(t))
$$

The solution to (1.1), for the given initial condition $y\left(t_{0}\right)=y_{0}$, is

$$
y(t)=y\left(t_{0}\right)+\int_{t_{0}}^{t} \frac{d \xi}{F(y(\xi))} .
$$

The logistic differential equation

$$
y^{\prime}(t)=y(t)(1-y(t))
$$

\footnotetext{
*Corresponding author

Email address: rami_thenat@yu.edu.jo (Rami AlAhmad)

doi: $10.22436 /$ jmcs.027.01.05
}

Received: 2021-11-03 Revised: 2021-11-25 Accepted: 2022-01-01 
represents a special case of (1.1). It's an autonomous ordinary differential equation with a wide range of engineering applications. For the initial condition $y(0)=1 / 2$, the logistic differential equation yields the solution

$$
y(t)=\frac{1}{1+e^{-t}} .
$$

Recent advancements in calculus have allowed for different presentations of the autonomous equation (1.1). In particular, the fractional representations of the autonomous differential equation, see [15]. Contrary to the Riemann-Liouville fractional derivative, the initial conditions are properly defined. The Caputo fractional derivative is defined as

$$
\left({ }_{a}^{C} D^{\alpha} f\right)(t)=\frac{1}{\Gamma(n-\alpha)} \int_{a}^{t} \frac{f^{(n)}(\tau) d \tau}{(t-\tau)^{\alpha+1-n}}
$$

where $n-1<\alpha \leqslant n$. Recently, the global impact of the corona virus (COVID-19) and epidemic models. In [11], the formulated fractional temporal SEIR, represents a state in which a disease is latent, measles model is given by:

$$
\begin{aligned}
& { }_{a}^{C} D_{t}^{\alpha} S=b-(\beta(t) I+\mu) S, \\
& { }_{a}^{C} D_{t}^{\alpha} E=\beta(t) S I-(s+\mu) E, \\
& { }_{a}^{C} D_{t}^{\alpha} I=\sigma E-(\zeta+\mu) I, \\
& { }_{a}^{C} D_{t}^{\alpha} R=\zeta I-\mu R .
\end{aligned}
$$

In this paper, we proceed to study the following fractional version of the autonomous differential equation (1.1):

$$
\left({ }^{C F} D^{\alpha} y\right)(t)=F(y(t)),
$$

where ${ }^{\mathrm{CF}} \mathrm{D}^{\alpha}$ is the Caputo-Fabrizio fractional derivative. We provide an example of a differential fractional version of the exponential growth function. Our findings are an extension to the results found in [15].

\section{Preliminaries}

Caputo and Fabrizio presented a definition of fractional derivative with a non singular kernel as follows. For a real smooth function $f$ and for $\alpha \in[0,1]$, the Caputo-Fabrizio fractional derivative [10] is given by

$$
\left({ }^{C F} D^{\alpha} f\right)(t)= \begin{cases}\frac{1}{1-\alpha} \int_{0}^{t} e^{-\frac{\alpha}{1-\alpha}(t-u)} f^{\prime}(u) d u, & 0 \leqslant \alpha<1, \\ f^{\prime}(t), & \alpha=1 .\end{cases}
$$

According to this definition, the following are the Caputo-Fabrizio fractional derivatives for some elementary functions.

Proposition 2.1. For $\mathrm{f}(\mathrm{t})=\mathrm{c}$, then $\left({ }^{\mathrm{CF}} \mathrm{D}^{\alpha} \mathrm{f}\right)(\mathrm{t})=0$.

Proposition 2.2. For $\mathrm{f}(\mathrm{t})=\mathrm{t}$, then $\left({ }^{\mathrm{CF}} \mathrm{D}^{\alpha} \mathrm{f}\right)(\mathrm{t})=\frac{1-e^{-\frac{\alpha}{1-\alpha}}}{\alpha}$.

Proposition 2.3. For $\mathrm{f}(\mathrm{t})=\mathrm{t}^{\mathrm{r}} ; \mathfrak{R}(\mathrm{r})>-1$, then $\left({ }^{\mathrm{CF}} \mathrm{D}^{\alpha} \mathrm{f}\right)(\mathrm{t})=\frac{1}{1-a} e^{-\frac{a t}{1-a}}\left(\frac{a}{1-a}\right)^{-r-1} \gamma\left(\mathrm{r}+1, \frac{\mathrm{at}}{1-\mathrm{a}}\right)$, where $\gamma$ is the incomplete gamma function, see $[1,2]$.

Proposition 2.4. For $f(t)=e^{b t}$, then $\left({ }^{C F} D^{\alpha} f\right)(t)=\frac{b\left(e^{\frac{a t}{a-1}}-e^{b t}\right)}{a(b-1)-b}$.

The following proposition gives the Laplace transform of the Caputo-Fabrizio fractional derivative. For advanced properties of Laplace transform, see [3]. 
Proposition 2.5. Let ${ }^{\mathrm{CF}} \mathrm{D}^{\alpha}$ be the Caputo-Fabrizio fractional derivative. The Laplace transform of $\left({ }^{\mathrm{CF}} \mathrm{D}^{\alpha} \mathrm{f}\right)(\mathrm{t})$ is $\frac{1}{\alpha+s(1-\alpha)}(s F(s)-f(0)) ; \Re(s)>\frac{\alpha}{\alpha-1}$, where $F(s)$ is the Laplace transform of $f(t)$.

Proof. Using (2.1), $g(t)=\left({ }^{C F} D^{\alpha} f\right)(t)$ satisfies

$$
(1-\alpha) e^{\frac{\alpha t}{1-\alpha}} g(t)=\int_{0}^{t} e^{\frac{\alpha u}{1-\alpha}} f^{\prime}(u) d u .
$$

Differentiating both sides of (2.2) and simplifying we get:

$$
(1-\alpha) g^{\prime}(t)+\alpha g(t)=f^{\prime}(t)
$$

Taking Laplace transform for both sides implies:

$$
(1-\alpha) s G(s)+\alpha G(s)=s F(s)-f(0),
$$

where $F(s)=L_{t}[f(t)](s)$ and $G(s)=L_{t}[g(t)](s)$. Now, solving (2.4) for $G(s)$, the result follows.

This result is read as

$$
\left({ }^{C F} D^{\alpha} f\right)(t)=L^{-1}\left[\frac{1}{\alpha+s(1-\alpha)}(s F(s)-f(0))\right],
$$

where $L^{-1}$ is the inverse Laplace transform.

Example 2.6. In this example, we solve a fractional version of the exponential growth (decay) differential equation $\mathrm{y}^{\prime}=\mathrm{ky}$. Consider the differential equation

$$
\left({ }^{C F} D^{\alpha} y\right)(t)=k y(t) .
$$

To solve this equation, take the Laplace transform for both sides of this equation to get:

$$
\frac{1}{\alpha+s(1-\alpha)}(s Y(s)-y(0))=k Y(s) \text {. }
$$

Solving this equation for $Y(s)$ implies that

$$
Y(s)=\frac{y(0)}{((\alpha-1) k+1) s-\alpha k}
$$

Therefore, the solution of $(2.5)$ is $y(t)=C e^{\frac{\alpha k t}{1-(1-\alpha) k}}$.

\section{The solution of the autonomous fractional differential equation}

Theorem 3.1. The solution of the autonomous fractional differential equation

$$
\left({ }^{C F} D^{\alpha} y\right)(t)=F(y(t))
$$

is given implicitly as

$$
\int_{y\left(t_{0}\right)}^{y(t)} \frac{d u}{F(u)}-(1-\alpha) \ln \left(\frac{F(y(t))}{F\left(y\left(t_{0}\right)\right)}\right)=\alpha\left(t-t_{0}\right) .
$$

Proof. Let $y(t)$ be a solution of (3.1). Then the definition (2.1) gives:

$$
\frac{1}{1-\alpha} e^{-\frac{\alpha}{1-\alpha} t} \int_{0}^{t} e^{\frac{\alpha}{1-\alpha} u} y^{\prime}(u) d u=F(y(t))
$$

Equivalently,

$$
\int_{0}^{t} e^{\frac{\alpha}{1-\alpha}} u y^{\prime}(u) d u=(1-\alpha) e^{\frac{\alpha}{1-\alpha} t} F(y(t))
$$


Differentiate both sides and simplifying we get:

$$
y^{\prime}(t)=(1-\alpha) F^{\prime}(y(t)) y^{\prime}(t)+\alpha F(y(t))
$$

Rearrange the terms as:

$$
\frac{y^{\prime}(t)}{F(y(t))}-(1-\alpha) \frac{F^{\prime}\left(y(t) y^{\prime}(t)\right)}{F(y(t))}=\alpha
$$

Integrating both sides from $t_{0}$ to $t$ we get:

$$
\int_{t_{0}}^{t} \frac{y^{\prime}(u)}{F(y(u))} d u-(1-\alpha) \int_{t_{0}}^{t} \frac{F^{\prime}\left(y(u) y^{\prime}(u)\right)}{F(y(u))} d u=\alpha \int_{t_{0}}^{t} 1 d u .
$$

Using integration by substitution for the integrals in the left side we get the desired result.

Example 3.2. To solve (2.5), use (3.2) to get that

$$
\int_{y\left(t_{0}\right)}^{y(t)} \frac{d u}{k u}-(1-\alpha) \ln \left(\frac{k y(t)}{k y\left(t_{0}\right)}\right)=\alpha\left(t-t_{0}\right)
$$

Therefore,

$$
\ln (y(t))-k(1-\alpha) \ln y(t)=k \alpha t+C
$$

Hence,

$$
(1-k(1-\alpha)) \ln (y(t))=k \alpha t+C .
$$

This implies the solution of (2.5) is

$$
y(t)=C e^{\frac{k \alpha t}{1-k(1-\alpha)}}
$$

It yields same result as in Example 2.6.

Example 3.3. We construct the solution of the fractional logistic differential equation and plot for different values of $\alpha$,

$$
\left({ }^{C F} D^{\alpha} y\right)(t)=y(t)(1-y(t))
$$

According to (3.2), the solution is implicitly given as

$$
\int_{y\left(t_{0}\right)}^{y(t)} \frac{d u}{u(1-u)}-(1-\alpha) \ln \left(\frac{y(t)(1-y(t))}{y\left(t_{0}\right)\left(1-y\left(t_{0}\right)\right)}\right)=\alpha\left(t-t_{0}\right)
$$

After simplifications, we will get:

$$
y^{\alpha}(1-y)^{\alpha-2}=C e^{\alpha t}
$$

where $C$ is a constant which depends on $\alpha$ and the initial conditions $t_{0}$ and $y\left(t_{0}\right)$. If we assume that $y(0)=1 / 2$, then the resulting solution is

$$
y^{\alpha}(1-y)^{\alpha-2}=4^{1-\alpha} e^{\alpha t}
$$

The set of solutions for different values of $\alpha$, along with the solution of the logistic differential equation (1.2) are shown by Figure 1. 


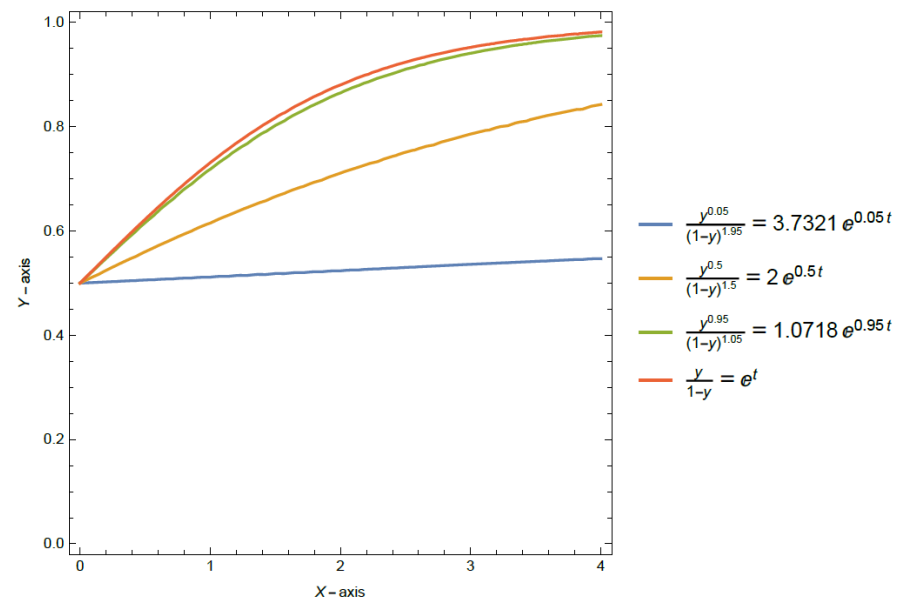

Figure 1: Solutions of the autonomous fractional differential equation (3.3) with $\alpha=0.05,0.5,0.95,1$.

Example 3.4. We construct a solution to the fractional autonomous differential equation

$$
\left({ }^{\mathrm{CF}} \mathrm{D}^{\alpha} \mathrm{y}\right)(\mathrm{t})=1+\mathrm{y}^{2}(\mathrm{t}) \text {. }
$$

According to (3.2), the solution is implicitly given as

$$
\int_{y\left(t_{0}\right)}^{y(t)} \frac{d u}{1+u^{2}}-(1-\alpha) \ln \left(\frac{1+y^{2}(t)}{\left.1+y^{2}\left(t_{0}\right)\right)}\right)=\alpha\left(t-t_{0}\right)
$$

After simplifications, we will get

$$
\arctan (y)-(1-\alpha) \ln \left(1+y^{2}\right)=\alpha t+C .
$$

If we assume that $y(0)=0$, then the resulting solution is

$$
\arctan (y)-(1-\alpha) \ln \left(1+y^{2}\right)=\alpha t
$$

The set of solutions for different values of $\alpha$, along with the solution of the differential equation $y^{\prime}=1+y^{2}$ are represented by Figure 2.

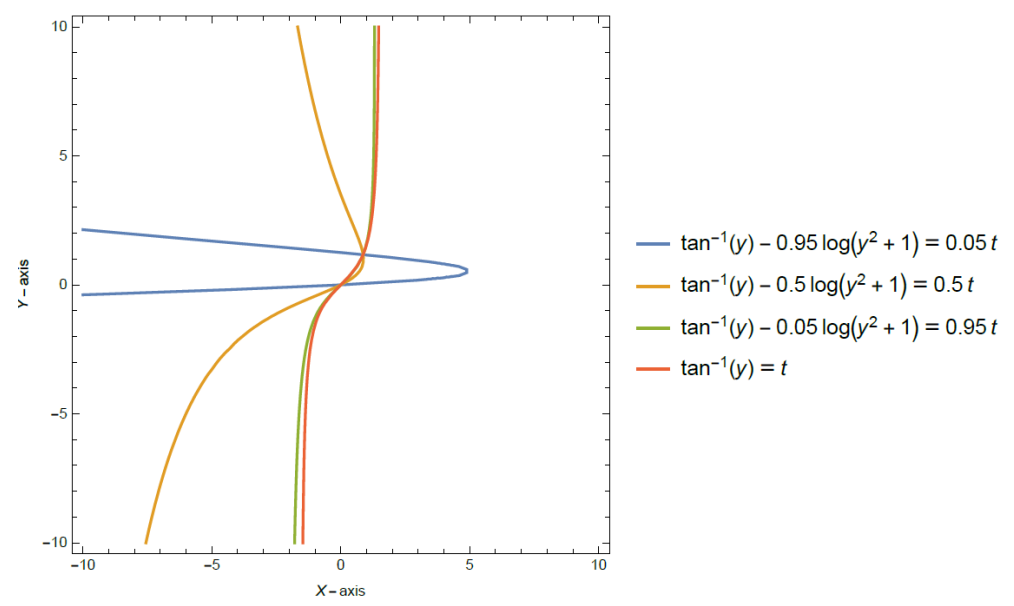

Figure 2: Solutions of the autonomous fractional differential equation (3.4) with $\alpha=0.05,0.5,0.95,1$. 


\section{Conclusion}

By using the Caputo-Fabrizio fractional derivative, we have proved that solutions can be obtained for different examples of the fractional autonomous differential equations. In addition, for initial conditions, we presented sets of solutions for different values of $\alpha$ between 0 and 1 . In particular, we arrived at the same solution as [15].

\section{References}

[1] R. AlAhmad, Products of incomplete gamma functions, Analysis (Berlin), 36 (2016), 199-203. 2.3

[2] R. AlAhmad, Products of Incomplete gamma functions Integral representations, Math. Sci. Appl. E-Notes, 4 (2016), 47-51. 2.3

[3] R. AlAhmad, Laplace transform of the product of two functions, Ital. J. Pure Appl. Math., 2020 (2020), 800-804. 2

[4] R. AlAhmad, M. Al-Jararha, On solving some classes of second order ODEs, Italian J. Pure Appl. Math., 45 (2021), 673-688. 1

[5] R. AlAhmad, M. Al-Jararha, H. AlMefleh, Exactness of second order ordinary differential equations and integrating factors, Jordan J. Math. Stat., 11 (2015), 155-167.

[6] S. Al-Ahmad, N. Anakira, M. Mamat, I. M. Suliman, R. AlAhmad, Modified differential transformation scheme for solving classes of non-linear differential equations, TWMS J. Appl. Eng. Math., (Accepted). 1

[7] S. Al-Ahmad, M. Mamat, R. Al-Ahmad, Finding Differential Transform Using Difference Equations, IAENG Int. J. Appl. Math., 50 (2020), 127-132.

[8] S. Al-Ahmad, M. Mamat, R. Al-Ahmad, I. M. Sulaiman, P. L. Ghazali, M. A. Mohamed, On New Properties of Differential Transform via Difference Equations, Int. J. Eng. Tech., 7 (2018), 321-324.

[9] R. AlAhmad, R. Weikard, On inverse problems for left-definite discrete Sturm-Liouville equations, Oper. Matrices, 7 (2013), 35-70.

[10] M. Caputo, M. Fabrizio, On the singular kernels for fractional derivatives. some applications to partial differential equations, Progr. Fract. Differ. Appl., 7 (2021), 79-82. 2

[11] Y. L. Chen, F. W. Liu, Q. Yu, T. Z. Li, Review of fractional epidemic models, Appl. Math. Model., 97 (2021), 281-307. 1

[12] R. K. Gazizov, S. Y. Lukashchuk, Higher-order symmetries of a time-fractional anomalous diffusion equation, Mathematics, 9 (2021), 11 pages. 1

[13] C. Ionescu, A. Lopes, D. Copot, J. A. T. Machado, J. H. T. Bates, The role of fractional calculus in modeling biological phenomena: a review, Commun. Nonlinear Sci. Numer. Simul., 51 (2017), 141-159. 1

[14] C. I. Muresan, I. R. Birs, E. H. Dulf, D. Copot, L. Miclea, A review of recent advances in fractional-order sensing and filtering techniques, Sensors, 21 (2021), 281-307. 1

[15] J. J. Nieto, Solution of a fractional logistic ordinary differential equation, Appl. Math. Lett., 123 (2022), 5 pages. 1, 1, 4

[16] H. R. Thieme, Mathematics in Population Biology, Princeton University Press, Princeton, (2018). 